\title{
A mixed-methods study of the implementation of medication adherence policy solutions: how do European countries compare?
}

This article was published in the following Dove Press journal:

Patient Preference and Adherence

27 October 2015

Number of times this article has been viewed

\author{
Wendy Clyne' \\ Sarah McLachlan² \\ 'Faculty of Health and Life Sciences, \\ Coventry University, Priory Street, \\ Coventry, UK; ${ }^{2}$ Primary Care and \\ Health Sciences, Keele University, \\ Staffordshire, UK
}

Objectives: We describe a key informant study that invited national medicines policy leads for the European Union member states to self-assess the level of implementation of medicines adherence initiatives in their country and the adequacy of that implementation. Interviews with medicines policy leads enabled in-depth understanding of the variation in adherence support across nations and the ways in which different nations prioritize, plan, and implement medicines adherence systems and services.

Methods: Ten national policy leads (Bulgaria, Denmark, Estonia, Finland, Germany, Ireland, Latvia, Lithuania, Malta, and the Netherlands) completed a self-assessment survey, and seven (Estonia, Finland, Germany, Ireland, Lithuania, Malta, and the Netherlands) engaged in a follow-up interview

Key findings: Policy leads varied in the level of implementation of medication adherence solutions that they reported in their nations; most initiatives were aimed directly at patients with few initiatives at government or health care commissioner levels of action. Policy leads reported insufficient implementation of medication adherence initiatives across all potential domains. Barriers to implementation included lack of resources, strategic planning, evidence to support action, the "hidden" nature of medication adherence within policy work, and dispersed responsibility for medication adherence as a policy and practice theme.

Conclusion: This study has international significance and summarizes the emergent characteristics of nations with and without coordinated medication adherence activity. We highlight the importance of sharing good practice in policy formulation and implementation for medication adherence.

Keywords: medication adherence, policy implementation, medicines policy, health care policy

\section{Introduction}

At any one time, $30 \%-50 \%$ of the European populations who are prescribed medication for the prevention or treatment of illness are nonadherent to that medication. ${ }^{1}$ Guidance exists to support health care providers and professionals deliver services that support medicines adherence, ${ }^{2}$ and there are many examples of current services that could be replicated and commissioned to support medicines adherence. ${ }^{3,4}$ Yet widespread adoption, commissioning, and implementation of medicines adherence support and services remain elusive. A challenge for health care commissioners and policymakers is deciding what can be commissioned and delivered in routine clinical practice and within limited budgets. Little is known about how policymakers and commissioners perceive the state of the art in medicines adherence support, the factors that influence how policymakers formulate relevant policies, or commissioners' perceptions of the extent and adequacy of current
Correspondence: Wendy Clyne Faculty of Health and Life Sciences, Coventry University, Priory Street, Coventry CVI 5FB, UK Email wendy.clyne@coventry.ac.uk 
policy implementation to support medicines adherence. There have been only limited attempts to understand how health care policymakers and commissioners utilize research evidence in this area, incorporate that evidence with other priorities, and make decisions about the nature, level, and type of input that may be needed and appropriate in their locality.

Here, we present a key informant study in which policymakers self-assess policy implementation for medication adherence in their own nation and discuss the range of influences on their policy decision-making.

The nature of the relationship between research and health care policy has been conceptualized in a number of ways. Traditional models propose a straightforward linear, rational process in which research knowledge is transferred to policymakers. Contextual and multidimensional models instead propose that the reductionist approach of linear models simplifies the nature of the relationship between research and policy and fails to take account of the broader range of influences on policymakers, the range of stakeholders involved in the policy influencing process, and the competing priorities that policymakers juggle. ${ }^{5-7}$

In addition, there are a number of specific factors in the medication adherence field that mitigate against a strong uptake of research evidence by policymakers. Research with policymakers demonstrates that they appreciate brevity and clear, simple messages, rather than academic-oriented publications to inform their views and actions. ${ }^{8}$ A number of terms, and concepts underpinning those terms, have been used to describe medication adherence. Multiple terms for similar concepts, and misuse of those terms, may hinder the nonspecialist policymaker from engaging with the adherence field and its literature. A range of theoretical and conceptual models have previously been used to explain the causes of nonadherence. Theoretical plurality benefits a burgeoning and growing research literature as common causes of nonadherence across a range of illnesses and medications are found and exceptions to the rule are identified. However, the hard-pressed policymaker may find diversity in the medication adherence narrative a barrier to action: how can we fix the problem of nonadherence if the experts cannot agree on what it is and what causes it?

Elsewhere, ${ }^{9}$ we report the development of medication adherence policy solutions by a panel of experts belonging to medicines stakeholder groups who took part in an online Delphi study and subsequent consensus meeting. This resulted in 26 consensus-based policy recommendations deemed by the panel to be high priority for addressing medication nonadherence across Europe (Table 1). The breadth of the policy solutions developed by the expert panel enables significant flexibility in local implementation, to reflect differences in health care systems, health-related culture, available resources, and the level and sophistication of existing implementation within nations and localities.

Here, we describe a subsequent study to explore the perceptions of medicines policymakers of the extent of implementation of those 26 consensus-based policy solutions within member states of the European Union. Specifically, we set out to explore the following:

1) The extent to which each of the 26 consensus-based policy solutions had been implemented in European countries.

2) Where countries have implemented one or more of the policy solutions, to obtain information on any benefits and costs resulting from implementation.

3) Perceptions of the appropriateness of current levels of implementation.

4) Where countries have not implemented the policy solutions, to determine whether the recommendations will feature in future planning to address nonadherence to medication.

5) Any perceived barriers to implementing the policy solutions within countries.

\section{Method}

\section{Participants}

Purposive sampling was used to identify the National Lead with responsibility for medicines policy, including adherence to medication, in each of the (then) 27 member states of the European Union. National Leads were contacted through correspondence with National Ministries of Health, National Medicines Authorities or Agencies, and representatives of the European Medicines Agency. National Leads who were unable to take part in the study were invited to nominate their deputies to participate (all participants are referred to as National Leads here). During the process of sampling, it became apparent that responsibility for policy on adherence was often distributed across various individuals, departments, and even authorities. In these cases, National Leads were invited to refer questions to colleagues if they did not feel well-placed to respond. All components of the study were conducted in the English language.

\section{Design}

A mixed-methods design was employed. A cross-sectional online survey was administered through Survey Monkey software in the first phase of the study, and semi-structured telephone interviews were conducted in the second phase. 
Table I The European consensus-based policy solutions for medicines adherence

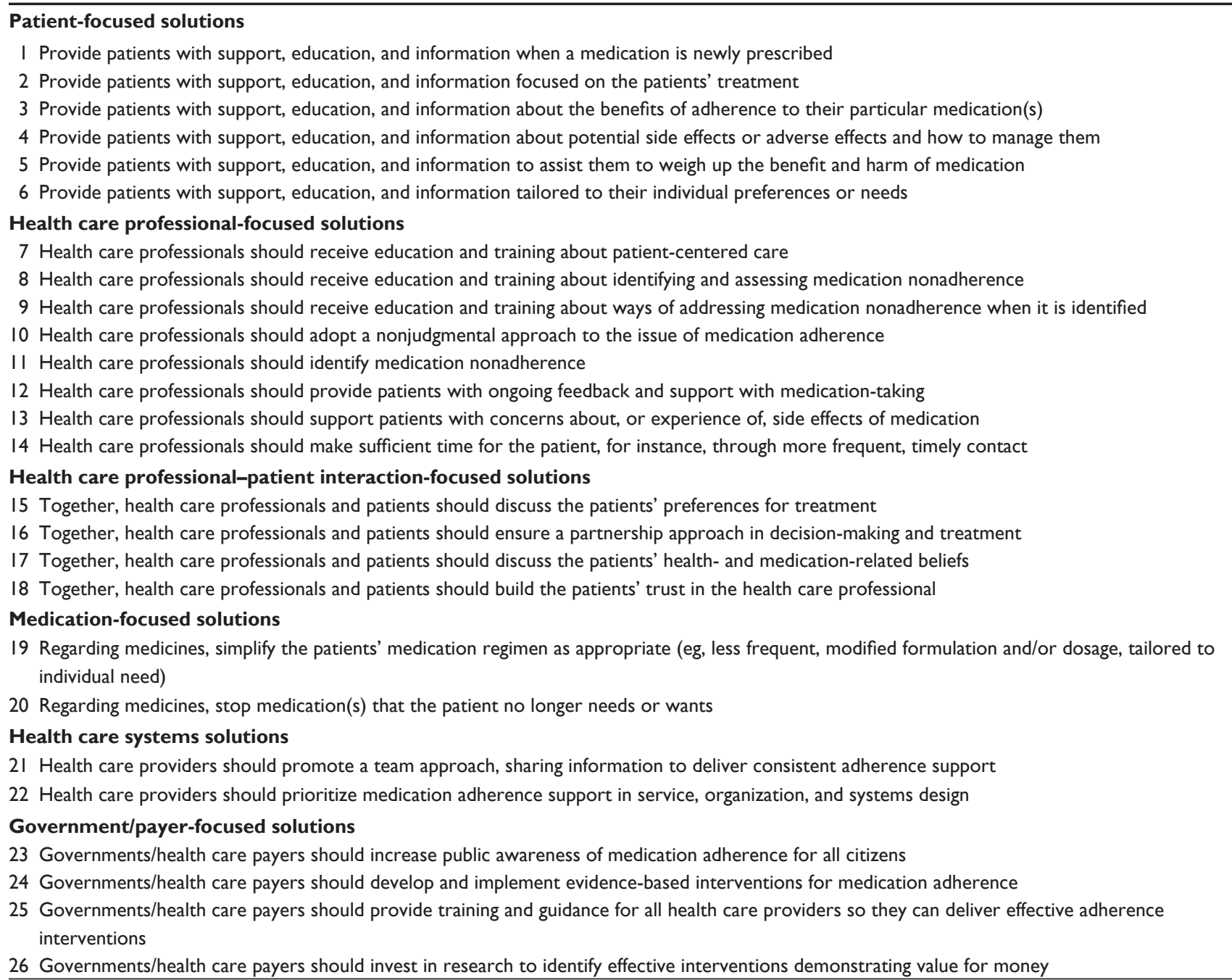

Note: $\odot 2012$ Clyne et al.; licensee BioMed Central Ltd. Adapted from Clyne W, White S, McLachlan S. Developing Consensus-Based Policy Solutions for Medicines Adherence for Europe: a Delphi Study. London: BMC Health Services Research; 2012.9 Creative Commons license and disclaimer available from: http://creativecommons.org/ licenses/by/4.0/legalcode

\section{Measures}

\section{Self-assessment survey}

The self-assessment survey was designed to enable quantitative assessment of each country's own policies on medication adherence in relation to the 26 consensus-based adherence policy solutions. Policy solutions were presented in the following categories, according to the main target of action: patient-focused, health care professional-focused, clinicianpatient interaction-focused, health-system-focused, and government-focused solutions as shown in Table 1.

Participants were asked to rate each policy solution on two scales. The first scale assessed the extent to which each policy solution had been implemented in the respondent's nation, using a five-point Likert-type response scale anchored by "discussed and considered but not implemented" (1) and "fully implemented in all regions for all health conditions" (5). The second scale measured participants' perceptions of appropriateness of the level of implementation for each policy solution. Ratings were made on a five-point Likert-type response scale anchored by "far too little implementation" (1) and "far too much implementation" (5). These scales were adapted from the medication self-assessment scale of the Institute for Safe Medication Practices. ${ }^{10}$

\section{Interview schedule}

The interview schedule was developed to explore participants' responses to the self-assessment survey and to identify examples of best practice for adherence to medication in each of the countries. A semi-structured approach was taken to ensure a degree of standardization across the interviews while also allowing participants to raise other issues, ideas, or concerns. ${ }^{11}$ Questions focused on eliciting examples of 
activities to support adherence within the various categories, participants' decision-making processes regarding implementation of particular policies, barriers to implementation, consequences of implementation, and future planning for medication adherence. Each participant was also asked about models of best practice for supporting adherence within their nation. Although a number of key questions were posed to each National Lead, for instance, regarding models of best practice for medication adherence, the interview schedule was tailored to each participant according to their survey responses. For instance, if a National Lead had indicated that a particular policy solution had been implemented within their country, a question on the benefits of implementation was incorporated within the interview schedule. Interviews proceeded systematically, addressing each category of policy solution in turn before moving to more general questions about best practices for medication adherence and impressions of the consensus-based policy solutions.

\section{Procedure}

Ethical approval for the study was secured from Keele University Ethical Review panel. National Leads were invited to participate in the study through email correspondence during early 2012. Each National Lead received a letter of invitation, a participant information sheet, a letter of support for the study from the Chief Pharmaceutical Officer of England, and a briefing document detailing the methods and results of the Delphi study. Prospective participants were invited to contact the researchers with any queries about the study or if they required any further information. All participants were informed that their anonymity could not be guaranteed because of the specificity of the sample. Participants were assured that they would be offered the opportunity to view drafts of any manuscripts containing their data and request amendments. Upon entering the survey, participants responded to three informed consent questions regarding their participation in the survey. Participants were also asked to indicate their consent to take part in a follow-up interview. Those who consented to engage in a follow-up interview were asked a series of questions about the recording of their interview and the use of quotations from interview transcripts.

Following the provision of consent, participants were presented with instructions for completing the self-assessment survey and proceeded to rate each policy solution. After completion of the survey, participants received a feedback document containing details of their ratings. Those who had consented to engage in a follow-up interview received a copy of the interview schedule and were asked to indicate their availability for interview. Where possible, interviews were conducted within 2 weeks of participants completing the survey. All interviews were conducted by two researchers over the telephone and lasted between 20 minutes and 1 hour. Six National Leads consented to the recording of their interviews, and one gave permission for detailed notes to be taken.

\section{Data analysis}

\section{Self-assessment survey data}

The quantitative data gathered through the survey were collapsed across categories of policy solution. Mean substitution was used to replace missing data. Summary descriptive data were produced for each category of policy solution in each country in order to draw cross-country comparisons on level of implementation and perceived appropriateness of implementation.

\section{Interview data}

The qualitative data comprised interview transcripts and detailed field notes. Each transcript or set of field notes was summarized and sent electronically to the appropriate National Lead. National Leads were asked to check that the summary provided an accurate reflection of the interview discussion and invited to add to or amend the content. The finalized and approved interview summaries were used as the basis for data analysis. Data analysis proceeded in an inductive and iterative fashion. Following the approach of Lavis et al, ${ }^{12}$ themes were identified using the constant comparative method of analysis. The researchers read interview transcripts as they became available and met regularly to discuss themes and issues arising from the data. A two-stage fragmenting and connecting procedure ${ }^{13}$ was employed during analysis. Initially, individual themes were extracted from the data. These themes were then compared both within and across interviews to explore the similarities and differences in services and provision between countries. In addition to facilitating comparisons between countries, this approach enabled modification of the interview schedule in light of new themes and ideas arising from the data.

\section{Results}

Of the 27 National Leads invited to participate in the study, ten (Bulgaria, Denmark, Estonia, Finland, Germany, Ireland, Latvia, Lithuania, Malta, and the Netherlands) completed the self-assessment survey and seven (Estonia, Finland, Germany, Ireland, Lithuania, Malta, and the Netherlands) engaged in a follow-up interview. 


\section{Survey data}

The mean total implementation rating for the 26 policy solutions across the ten participating countries was 77.97 ( $\mathrm{SD}=10.38$; range $=26-130$ ). Bulgaria attained the highest overall level of self-assessed implementation, and Estonia provided the lowest total implementation rating (Figure 1). The mean implementation scores across countries for each category of policy solution (Figure 2) indicated that most implementation had taken place at the patient level including, for example, the provision of support, education and information about newly prescribed medicines, and the benefits of adherence. This was followed closely by activity focused on improving patient-clinician interactions, for instance, through the implementation of a partnership approach. The lowest amount of activity was reported for policy solutions at the government or health care payer level, which included investment in research to identify effective interventions demonstrating value for money and increasing public awareness of medication adherence. For all six categories of policy solutions, the mean rating for perceived appropriateness of level of implementation fell $<3.00$, indicating that across the ten European countries in the survey, National Leads felt insufficient implementation had taken place for medication adherence in all policy areas.

The mean level of implementation and perceived appropriateness of implementation for each category of policy solution are shown for each of the ten countries in Figure 3. The mean ratings of perceived appropriateness of implementation are below the midpoint for 52 of the 60 scores, suggesting that the majority of National Leads felt that more could or should be done to support patients with adherence to medication within their nations, across target areas.

\section{Interview data}

A range of themes around medication adherence emerged from the interview data, and the majority of themes were present across the data of two or more interviewees. Outlines of these themes are presented below. Key differences between nations with high and low levels of implementation activity are shown in Figure 4.

\section{Responsibility for medicines adherence policy and planning: meta level}

Four nations (Finland, Germany, Malta, and the Netherlands) described shared responsibility for adherence policy and planning at the higher level. The particular parties involved differed between the nations. Within the Netherlands, for example, the role of the government was described as a systems approach: ensuring good openings for adherence within the health system and removing obstacles. The policy lead in this nation explained that activity was implemented on a regional basis, as solutions were differentially effective in different regions. Health care insurers were portrayed as well placed, to influence the behavior of health care providers and patients with regard to adherence. An official working group for adherence, consisting of doctors, nurses, pharmacists, patients, insurers, and researchers, was seen as integral to adherence implementation in the Netherlands.

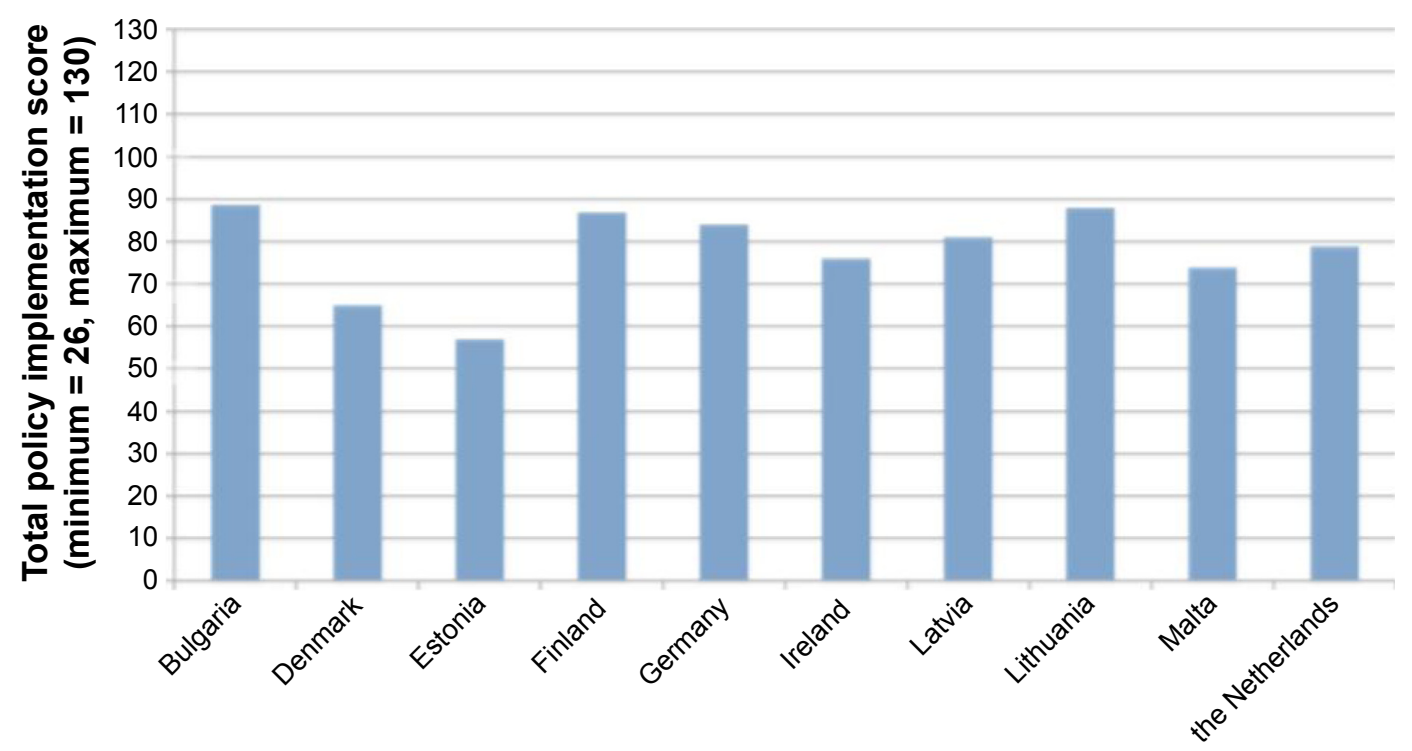

Figure I Total policy implementation score for each country. Note: Minimum possible score $=26$; maximum possible score $=130$. 


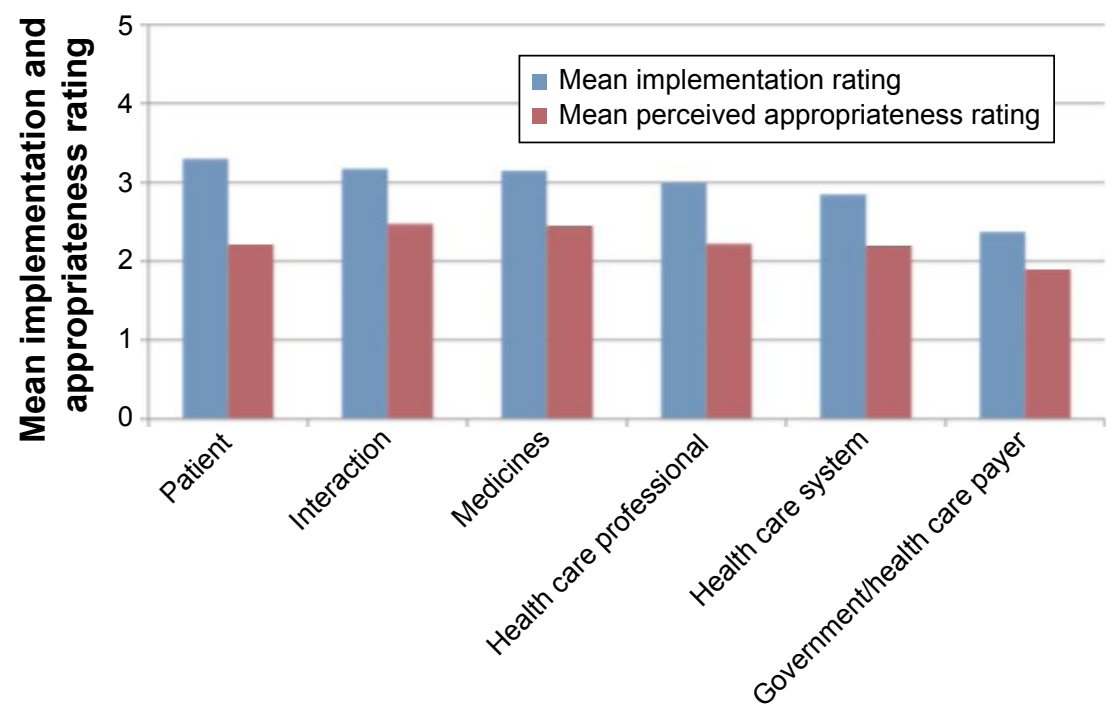

Figure 2 Mean implementation and perceived appropriateness of implementation across country for each category of policy solution.
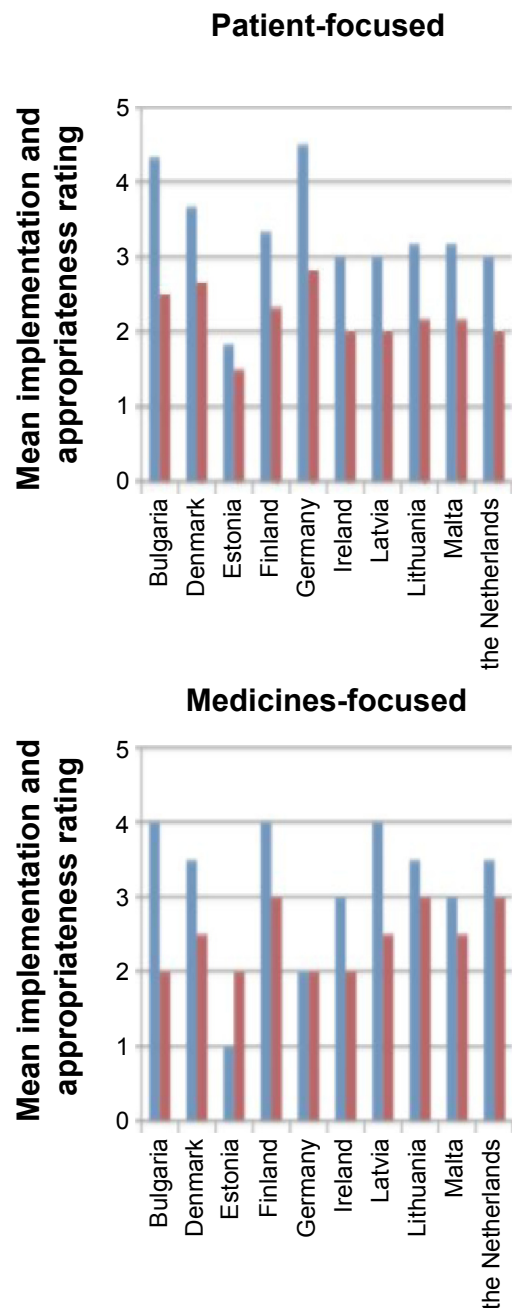

\section{Health care professional-focused}

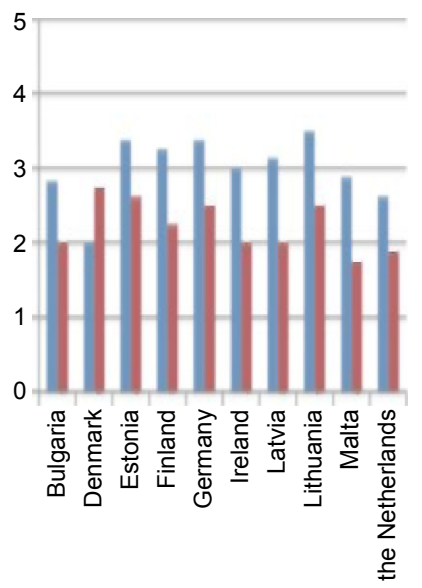

System-focused

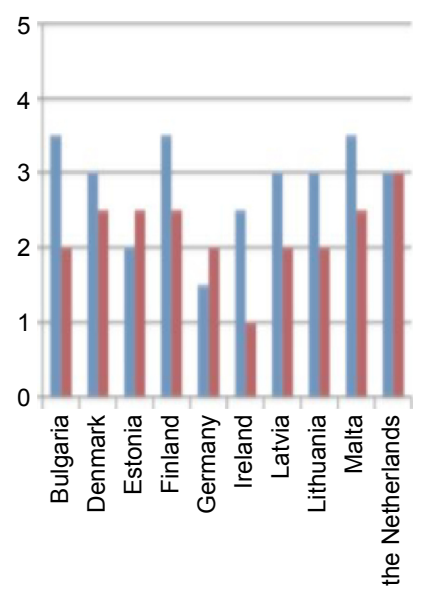

Interaction-focused

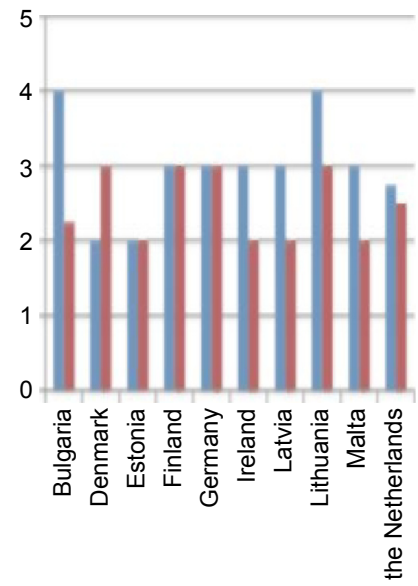

Government-focused

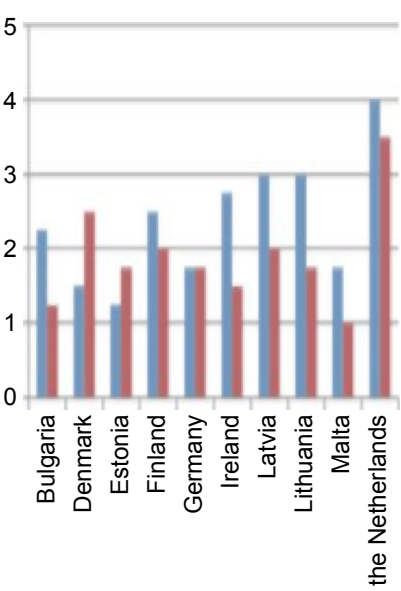

Mean perceived appropriateness rating $\quad$ Mean implementation rating

Figure 3 Mean implementation and perceived appropriateness of implementation of policy solutions for medication adherence, by category and country. 


\begin{tabular}{|ll|}
\hline $\begin{array}{l}\text { Exemplar of a nation with more activity for } \\
\text { adherence }\end{array}$ & $\begin{array}{l}\text { Exemplar of a nation with less activity for } \\
\text { adherence }\end{array}$ \\
\hline $\begin{array}{ll}\text { - Identification of medication adherence as } \\
\text { a priority }\end{array}$ & $\begin{array}{l}\text { - Medication adherence not identified as a } \\
\text { priority }\end{array}$ \\
- Structured policy forum for adherence & - No structured policy forum for policy on \\
- Involvement of all stakeholder groups & adherence \\
- History of activity for adherence & - Other issues prioritized above adherence \\
- Government investment in initiatives for & - No government investment in activity for \\
adherence & adherence \\
- Culture of patient-centered care & - Health care provider-centered approach \\
\hline
\end{tabular}

Figure 4 Comparison of an exemplar nation with more activity for adherence with exemplar of nation with less activity.

\section{Absence of adherence "theme" in policy documents and practice}

Six interviewees emphasized the "hidden" nature of adherence in both policy and practice. Several interviewees stated that policy documents in their nations did make reference to adherence, but these references were often distributed among a number of other topics, such as patient safety, rather than falling under the discrete label of "medication adherence". This reduced the visibility of medicines adherence as a focus in policy activity. Two of the policy leads indicated that the area of medication adherence was overshadowed by more pressing issues, such as the availability of medicines, or subject to competition for funding from other medicinesrelated topics.

The policy lead for adherence in the Netherlands explained that the decision to invest in services for adherence had been simple and cited the World Health Organization's ${ }^{1}$ report on adherence to long-term therapies as a prompt.

It was really quite simple because there was this WHO report about adherence ... and it said that about $50 \%$ of chronically ill patients didn't use their medicines as they were supposed to be used and when you see how much we pay for medicines in the healthcare system and then you say well, we throw away $50 \%$ of this; that's quite a sum! ... Yes, and that's really what made the case here to have investments in this area. And we still invest in this area, but on a nationwide scale, not a regional or local one. [the Netherlands respondent, 97-107]

In two other countries, the case for investment was less straightforward. Interviewees in Estonia and Germany indicated that a lack of money and resources presented significant barriers to implementing initiatives for adherence, even when strong proposals were in place.

\section{Evaluating options and assessing outcomes}

Three interviewees described difficulties in deciding which particular initiatives to implement. The policy lead for the Netherlands stated that some initiatives have clearly deserved government support, while decisions for other initiatives were less clear-cut. Issues relevant to these decisions included the scope of expected benefit in terms of widespread or localized effect, the expense of the initiative, and support from doctors or pharmacists. Interviewees in Estonia and Finland emphasized the need for research to identify effective and cost-effective interventions.

Interviewees for Finland, Germany, Ireland, Lithuania, and the Netherlands outlined the complexities of evaluating outcomes or benefits of initiatives for adherence. Specific problems included establishing objective, relevant, and independent assessment methods. Barriers to evaluation were discussed, such as shortages in time, resources, skills, and other competing priorities. A lack of studies evaluating the outcomes of initiatives was also raised as a problem. The policy lead for Finland highlighted the difficulty involved with identifying improvements in medication adherence, particularly as improvements in health outcomes cannot necessarily be attributed to increased adherence.

Variability in provision and the targeting of resources Policy leads in Finland, Lithuania, and Malta described variability, both planned and unplanned, in provision for medication adherence across different areas of practice and patient groups. In Finland, adherence was reported to be addressed more thoroughly in patients with long-term conditions, such as cardiac disease, diabetes, and asthma, as these patients tend to meet with their doctors more frequently than other patients. The policy lead for adherence in Malta contrasted areas of excellence, for instance, specialist teams where there 
is good communication between health care professionals and patients, strong collaboration between various health care professions, and detailed information available on the patient's history, with the general system, in which time and support for the patient are more limited.

Interviewees in Finland, Ireland, Malta, and the Netherlands referred to the targeting of resources and services. One strategy for targeted adherence support was a focus on patient groups with the more prevalent long-term conditions, for example, diabetes and asthma. Targeted support for adherence in the Netherlands focused particularly on conditions where medicines adherence can be more difficult, such as schizophrenia and asthma. Interviewees in Ireland and Malta reported the targeting of some initiatives, for example, medication review in Ireland, to particular clinical areas or to patients on complex regimes and/or with comorbidities. The targeting of services toward patients prescribed certain medicines, such as those needing regular monitoring, was also detailed by the policy lead for Malta.

\section{Barriers to implementation}

Interviewees mentioned a number of barriers to the implementation of policy solutions for medication adherence. Some of these barriers were common to several countries. A lack of resources and difficulty with financing activity for adherence were reported for Estonia, Finland, and Ireland. The need to ensure acceptance of the policy solutions and openness to patient-centered approaches were mentioned by interviewees for Estonia and Malta. Other barriers to implementation included delay and procrastination, shortages in doctors and health care centers, the difficulty of implementing best practice developed in one region into another region, and a lack of awareness in the general public with regard to aspects of medicines use. The challenge of achieving a balance between enforcement of policy and practicable implementation was described by the policy lead for Malta.

\section{The impact of major health system changes on adherence}

Three interviewees described the indirect effects of broad, health system reforms on implementation of medicines adherence initiatives. Major changes to the health care systems over recent years were described for each of these countries. Reforms in the Netherlands included increasing the role of health care insurers in designing pharmaceutical care. A radical overhaul of the health care system was reported for Ireland, and effects were described as filtering down to impact a variety of domains, including adherence.
Reforms in this nation also affected regulatory bodies and the education and training of health care professionals. The policy lead in Malta indicated that organizational changes offered a good opportunity to implement new standards for the use of medicines. The policy lead for Ireland referred to European Union initiatives, such as requirements for patient information, which may positively impact support for adherence in individual nations.

\section{Responsibility for adherence - patient level}

Consistent with interviewees' comments regarding responsibility and planning at the higher level, responsibility for adherence-focused activity at the patient level was also reported as shared across a number of stakeholders. The policy lead in the Netherlands emphasized the responsibility of "the triangle" - doctors, pharmacists, and patients - in making progress on adherence. Interviewees in Finland, Germany, Ireland, Lithuania, and Malta also referred to obligations on doctors and pharmacists to produce and deliver patient information, not restricted to but including that on adherence. The involvement of patient organizations in the provision of patient information was outlined by the policy leads for Estonia, Finland, Germany, and Malta, while the leads for Estonia and Finland additionally acknowledged the role played by the pharmaceutical industry in funding or collaborating with patient organizations. The policy lead for Finland also mentioned the importance of expert nurses in assisting patients with adherence to medication.

\section{Health care professionals' training and education}

Policy leads in Finland, Ireland, and Malta indicated that training and education on adherence to medication in their nations were especially well developed for pharmacists. In addition to content on adherence within the curricula of pharmacy courses, the National Lead for Malta explained that adherence is also covered within voluntary continuing professional education offered by the College of Pharmacy Practice. The interviewee in Ireland described a heavy emphasis on medication adherence in the training of pharmacists, particularly with regard to antibiotics, antidepressants, and antirejection therapy. Finland's policy lead reported a drive toward campaigns to educate pharmacists on how to improve medication adherence.

The policy leads in Germany, Ireland, and Malta all referred to the role of continuing professional development in education and training on adherence. Practice learning under the supervision of a tutor was also mentioned as occurring within Ireland. The interviewees in Germany and Ireland, 
as well as Lithuania, discussed health care professionals' training in methods that may promote adherence, such as patient-centered care and developing a partnership approach with patients. Interviewees also outlined some recent advances in training and education on adherence, such as doctors and nurses taking more credits on aspects of medicines use in Malta and the development of educational programs for doctors in Lithuania. The policy lead in Estonia stated that no research had been conducted on support for health care professionals in addressing patients' nonadherence, so the extent to which this support is provided was unknown.

\section{Partnership approach}

A partnership approach between patients and health care professionals was reported as implicit within the health systems of three countries: Germany, Ireland, and Malta. Policy leads in these countries indicated that health care professionals are aware of the importance of implementing a partnership approach. The interviewee for Germany commented that doctors and pharmacists may not explicitly discuss the need for a partnership approach with patients, but are nonetheless aware of the need to use such an approach.

Interviewees representing Ireland and Malta suggested that the health system culture in their nations now served to promote a partnership approach with patients, through the transition from a more paternalistic situation to one in which patients are able to participate more actively in decisions about their medicines.

\section{Interprofession collaboration}

Collaboration between professions in addressing patients' nonadherence was reported to varying degrees in Germany, Ireland, and the Netherlands. While the policy lead in the Netherlands described an established system of collaboration between pharmacists and doctors at the state level to ensure that information provided to patients is consistent, the policy lead in Germany mentioned proposed activities for improving the coworking between pharmacists and doctors within an action plan for drug safety. This interviewee acknowledged the need to improve cooperation, not only from the perspective of drug safety but also in a more general way. The policy lead for Ireland stated that steps had been taken toward promoting a collaborative approach between health care professions. For instance, the Health Service in Ireland pays pharmacists a nondispensing fee for medication prescribed but not dispensed, as an incentive to encourage collaboration between medical practitioners and pharmacists about the appropriateness of medicines.

\section{The role of technology in adherence}

Policy leads in Ireland, Lithuania, Malta, and the Netherlands described the abundance of objective information about medicines on the internet, including summaries of product characteristics and patient information leaflets. However, the use of this information by patients was unknown. The interviewee for the Netherlands explained that technology was being utilized to develop a nationwide monitor on adherence to assess whether initiatives to increase adherence have resulted in improved use of medicines. This monitor will allow comparisons to be made between different diseases and regions. Electronic systems to collate and share information on dispensing of prescriptions were discussed by the interviewees for Estonia and Lithuania. A digital system containing histories of patients' medicines is used by general practitioners to infer patients' adherence in Estonia, while an electronic prescribing system is under development in Lithuania. The policy lead in Ireland stressed the importance of health information technology in facilitating the sharing of information to deliver consistent adherence support, increasing public awareness of adherence, and enabling health care professionals to spend more time with patients. Such technology was described as having the potential to improve practice and produce a more cost-efficient health system.

\section{Advice for other nations}

With regard to advice for other nations, cooperation between stakeholders was described as particularly important. The interviewees for Finland, Malta, and the Netherlands referred to the need to engage all parties, such as patients, pharmacists, doctors, and government, in the planning and implementation of activity for adherence. Other recommendations offered by these countries included recognizing adherence as a problem to be addressed, striving for national-level coordination in initiating activity, and using clear treatment guidelines to facilitate standardization. The policy lead for Malta also suggested the targeting of interventions to those areas that would result in the greatest benefit, both financially and in terms of patient outcomes.

\section{Comprehensiveness of the policy solutions}

Six interviewees were asked about the comprehensiveness of the policy solutions, and all felt that they provided a complete account of the activity needed to address nonadherence. None of the interviewees suggested additional solutions.

\section{Discussion}

European medicines policy leads differ in their perceptions of the extent to which policies to support medication 
adherence have been implemented in their own countries. Policy leads reported that more implementation had taken place for solutions at the patient, patient-clinician interaction, and medicine levels than solutions at government or health care payer levels of action. In general, across the ten countries, implementation of medication adherence policy solutions was perceived to be insufficient. Medicines policy leads noted that medication adherence has limited visibility within policy documents, can be overshadowed by other health policy issues, and that difficulty demonstrating impact makes it harder to make a case for investment in adherence support. Countries with more successful implementation have a number of characteristics: coordinated multi-stakeholder forums, national-level support and drive, and a patientcentered approach to health care.

This is the first study we are aware of to examine the extent of implementation of medication adherence policy solutions and to do so across a number of countries. Though the sample size is lower than desired, it includes medicines policy leads from Western, Central, and Eastern Europe and from Northern and Southern Europe. In the absence of medication adherence outcome indicators, or benchmarks for medication adherence support, key informant interviews with medicines policy leads are an effective method for exploring the factors that influence how options for medication adherence implementation are formulated and the factors that determine the nature and level of implementation. Furthermore, as the subjective beliefs and opinions of policymakers are likely to impact on policy decisions and the allocation of resources, ${ }^{14}$ the ways in which policymakers perceive and understand the nature of medication nonadherence and the potential policy options for supporting medication adherence are of clear interest.

Social desirability bias to the survey may have led respondents to report more medication adherence implementation than is actually the case, to give a favorable impression of health service provision in their country. Several factors should be taken into account here. First, all respondents were potentially exposed to social desirability bias, yet varying perceptions of the level of implementation were reported. Seven of the ten respondents participated in follow-up interviews, and responses to the survey were discussed in detail. Participants would likely have encountered difficulty discussing and exploring their survey responses during interview if those responses were fallacious. Also, many respondents reported that the implementation of specific policy solutions was insufficient, an unlikely response if participants were weighted by a heavy social desirability bias.
The medicines policy leads described the implementation of medication adherence policy, in general, to be less than ideal and described a number of factors that impede them from formulating and implementing policy solutions in this area. Given the multidimensional nature of medication adherence and the way in which responsibility for medication adherence cuts across health care professional groups and sectors, and indeed across departments and roles in Ministries of Health, the low level of action reported at the systems and government levels is a concern. Examples of action at these levels were reported by a minority of nations, for example, the use of multi-stakeholder national forums and a policy drive to address medication adherence, and serve as models for other countries struggling to implement policy solutions in this area. The need to raise the profile of medication adherence in health policy formulation, reported to be hidden or invisible in policy documentation in many countries, also emerges as a priority for the medication adherence community.

In the majority of countries surveyed, activity to enhance or support medication adherence was rarely described as coordinated or part of a larger strategic policy program but seemed instead to emerge in a more ad hoc fashion and be focused at interventions aimed at modifying or supporting individual patient behavior. When activity was planned, it was also often targeted. Two main targeting strategies emerged: a focus on high prevalence long-term conditions, such as diabetes and asthma, and a focus on patients prescribed medicines with an element of complexity such as a requirement for additional monitoring for safety purposes or medicines that are known to be more problematic. The relative efficacy of these two strategies for enhancing medication adherence is unknown.

Several factors mentioned by the medicine leads hinder productive policymaking in this area and are also less amenable to rapid change. The medicines policy leads were short of evidence for the clinical and cost-effectiveness of intervening to address medication adherence, making it difficult for them to build a strong case for investment. Only one country - the Netherlands - reported that the prima facie evidence of the size of the problem of medication adherence and the implicit consequences of nonadherence for morbidity and mortality were sufficient in themselves to stimulate government-level action. In the medium to long term, it would seem likely that convincing evidence of the cost and clinical benefits of medication adherence support, well communicated to policymakers, will be necessary to stimulate concerted action to address medication adherence. 


\section{Conclusion}

This study used medicines policy leads from a number of European countries as key informants to understand the selection, implementation, and barriers to implementation of policies addressing medication adherence. National policy leads felt insufficient implementation of medication adherence policy solutions had taken place in all potential target areas. We demonstrate that while European countries differ in some key ways, such as the extent to which patient-centered care is dominant in health care culture and the existence of structured policy forums for medication adherence, medicines policy leads experienced similar difficulties and challenges in implementing medication adherence policy solutions and shared similarities in the nature of successful implementation. This suggests that coordinated action between countries at European level and the sharing of good practice in medication adherence policy formulation and policy implementation may be beneficial.

\section{Acknowledgments}

We are grateful to the medicines policy officials, named and anonymous, for their participation in the study: Girtel Liidemann, Ministry of Social Affairs, Estonia; Professor Maria Orbetzova, Sv. Georgy University Hospital, Medical University, Plovdiv, Bulgaria; Dr Patricia Vella Bonanno, Medicines Authority, Malta; Lidwein Verweij, Ministry of Health, Welfare and Sport, the Netherlands. This study, as part of the $\mathrm{ABC}$ project, was funded by the European Commission Seventh Framework Programme (FP7 Theme Health, 2007-3.1-5, grant agreement number 223477). The final project report is available here: http://abcproject.eu/ img/ABC $\% 20$ Final.pdf

\section{Authors' contribution}

WC was the principal investigator and responsible for the conception of the study. Both authors undertook the design and development of the survey and interview schedule. Both authors conducted the interviews, and SM transcribed the interviews. Both authors conducted the analysis and writing of the final manuscript. All authors contributed toward data analysis, drafting and critically revising the paper and agree to be accountable for all aspects of the work.

\section{Disclosure}

The authors declare that they have no competing interests.

\section{References}

1. World Health Organisation. Adherence to Long Term Therapies: Evidence for Action. Geneva: WHO; 2003.

2. Available from: http://www.nice.org.uk/cg76. Accessed March 23, 2015.

3. nice.org [homepage on the internet]. London: National Institute for Health and Care Excellence. Available from: http://www.nice.org.uk/ $\mathrm{mpc} /$. Accessed March 23, 2015.

4. Available from: http://psnc.org.uk/services-commissioning/servicesdatabase/. Accessed March 23, 2015.

5. Davies P. Is Evidence-Based Government Possible? [article on the Internet]. London; 2004 [cited May 28, 2012]. Available from: http://www.odi.org.uk/rapid/events/impact_insight/docs/jerry_lee_ lecture_12.02.04.pdf. Accessed March 23, 2015.

6. House of Commons Science and Technology Committee. Scientific Advice, Risk and Evidence Based Policy Making [article on the Internet]. London: House of Commons, 2005 [cited May 28, 2012]. Available from: http://www.publications.parliament.uk/pa/cm200506/ cmselect/cmsctech/900/900-i.pdf. Accessed March 23, 2015.

7. Dobrow MJ, Goel V, Upshur REG. Evidence based health policy: context and utilization. Soc Sci Med. 2004;58:207-217.

8. Ettelt S, Mays N. Health services research in Europe and its use for informing policy. J Health Serv Res Policy. 2011;16(Suppl 2): S48-S60.

9. Clyne W, White S, McLachlan S. Developing Consensus-Based Policy Solutions for Medicines Adherence for Europe: a Delphi Study. London: BMC Health Services Research; 2012.

10. Institute for Safe Medication Practices. ISMP Medication Safety Self Assessment ${ }^{\circledR}$ for Hospitals [Internet]. Canada: ISMP; 2011 [cited May 28, 2012]. Available from: http://www.ismp.org/selfassessments/ Hospital/2011/full.pdf. Accessed March 23, 2015.

11. Rapaport J, Manthorpe J, Hussein S, Moriarty J, Collins J. Old issues and new directions: perceptions of advocacy, its extent and effectiveness from a qualitative study of stakeholder views. J Intellect Disabil. 2006; 10:191-210.

12. Lavis JN, Oxman AD, Moynihan R, Paulsen EJ. Evidence-informed health policy 3 - Interviews with the directors of organisations that support the use of research evidence. Implement Sci. 2008;3:55.

13. Boeije $H$. A purposeful approach to the constant comparative method in the analysis of qualitative interviews. Qual Quant. 2002;36:391-409.

14. Blendon RJ, Steel Fisher GK. Commentary: understanding the underlying politics of health care policy decision making. Health Serv Res. 2009; 44:1137-1143.

\section{Publish your work in this journal}

Patient Preference and Adherence is an international, peer-reviewed, open access journal that focuses on the growing importance of patient preference and adherence throughout the therapeutic continuum. Patient satisfaction, acceptability, quality of life, compliance, persistence and their role in developing new therapeutic modalities and compounds to optimize clinical outcomes for existing disease states are major areas of interest for the journal. This journal has been accepted for indexing on PubMed Central. The manuscript management system is completely online and includes a very quick and fair peer-review system, which is all easy to use. Visit http://www. dovepress.com/testimonials.php to read real quotes from published authors. 